\title{
Quantum-classical correspondence of a field induced KAM-type transition: A QTM approach
}

\author{
P K CHATTARAJ*, S SENGUPTA and S GIRI \\ Department of Chemistry and Centre for Theoretical Studies, Indian Institute of Technology, \\ Kharagpur 721302 \\ e-mail: pkc@chem.iitkgp.ernet.in
}

\begin{abstract}
A transition from regular to chaotic behaviour in the dynamics of a classical Henon-Heiles oscillator in the presence of an external field is shown to have a similar quantum signature when studied using the pertaining phase portraits and the associated Kolmogorov-Sinai-Lyapunov entropies obtained through the corresponding Bohmian trajectories.
\end{abstract}

Keywords. Quantum potential; Bohmian trajectories; Henon-Heiles oscillator; KAM transition.

\section{Introduction}

Many classical non-linear dynamical systems exhibit Kolmogorov-Arnold-Moser (KAM) transition $^{1-5}$ wherein the dynamics of the system changes from a regular to a chaotic one by varying the non-integrability parameter present in the Hamiltonian. A quantum variant of the KAM transition is the subject matter of current research interest. ${ }^{6-17}$ Classical and quantum Henon-Heiles oscillators have been extensively studied for this purpose. ${ }^{5,18}$ Various diagnostics like the initially close Bohmian trajectories and the associated Kolmogorov-Sinai-Lyapunov (KSL) entropies, phase portraits and phase space volume (uncertainty product), autocorrelation function and the related power spectrum, etc. have been used ${ }^{18}$ in analysing the quantum domain behaviour of the classically chaotic Henon-Heiles oscillator as the degree of the non-linearity and non-integrability is increased.

In the present work, we start with the classical Henon-Heiles oscillator at the regular domain and expose it to an external field. As the intensity of the external field is increased it is expected that the oscillator dynamics will move from regular to chaotic as was noticed by Lin and Balentine ${ }^{19}$ in the field induced barrier penetration in a double-well oscillator as well as in its quantum analogue. ${ }^{20}$

Quantum potential based approaches like quantum fluid dynamics (QFD) ${ }^{21}$ and quantum theory of motion $(\mathrm{QTM})^{22}$ have been used in the past for understand-

\footnotetext{
*For correspondence
}

ing the quantum domain behaviour of these systems. ${ }^{18,20,22-24} \mathrm{We}$ make use of quantum potential based approaches in the present work to analyse the quantum analogue of the field induced KAM transition in a Henon-Heiles oscillator.

Sections 2 and 3 provide the theoretical background and the numerical technique adopted here. Results and discussion are presented in $\$ 4$ while $\$ 5$ contains some concluding remarks.

\section{Theoretical background}

The classical dynamics of the Henon-Heiles oscillator in presence of an axial external field applied in the $y$-direction is studied by solving the pertinent classical Hamilton's equations of motion with the following Hamiltonian:

$$
\begin{aligned}
H(x, y)= & \frac{1}{2}\left(p_{x}^{2}+p_{y}^{2}\right)+\frac{1}{2}\left(x^{2}+y^{2}\right) \\
& +\lambda x\left(y^{2}-\frac{x^{2}}{3}\right)-g y \cos (\omega t)
\end{aligned}
$$

where the first term stands for the kinetic energy, the second and the third terms respectively represent the harmonic and anharmonic parts of the potential energy of the oscillator whereas the last term takes care of the interaction of the oscillator with the external axial field. The non-linearity and the non-integrability parameter $\lambda$ has a value of $0 \cdot 1118034^{5,8}$ and the system quantization is performed with $\hbar=1, m=1$.

The associated time-dependent Schrödinger equation (TDSE) is given by 


$$
\begin{aligned}
& {\left[-\frac{1}{2}\left(\frac{\partial^{2}}{\partial x^{2}}+\frac{\partial^{2}}{\partial y^{2}}\right)+\frac{1}{2}\left(x^{2}+y^{2}\right)\right.} \\
& \left.+\lambda x\left(y^{2}-\frac{x^{2}}{3}\right)-g y \cos (\omega t)\right] \psi(x, y, t) \\
& =i \frac{\partial \psi(x, y, t)}{\partial t} ; i=\sqrt{-1}
\end{aligned}
$$

The wavefunction $\psi(x, y, t)$ may be written in the following polar form in terms of the density, $\rho(x, y$, $t$ ) and the velocity potential, $\chi(x, y, t)$ :

$$
\psi(x, y, t)=\rho^{1 / 2}(x, y, t) \exp [i \chi(x, y, t)]
$$

and the velocity components may be defined as

$$
\dot{x}=\left.\nabla_{x} \chi(x, y, t)\right|_{x=x(t)},
$$

and

$$
\dot{y}=\left.\nabla_{y} \chi(x, y, t)\right|_{y=y(t)}
$$

According to the quantum theory of motion (QTM), ${ }^{22}$ the quantum (Bohm) trajectories may be generated by solving (4) with different initial conditions. A phase space distance function has been defined as, ${ }^{18,20,23}$

$$
\begin{aligned}
D(t) & =\left\{\left[x_{1}(t)-x_{2}(t)\right]^{2}+\left[y_{1}(t)-y_{2}(t)\right]^{2}\right. \\
& \left.+\left[p_{x_{1}}(t)-p_{x_{2}}(t)\right]^{2}+\left[p_{y_{1}}(t)-p_{y_{2}}(t)\right]^{2}\right\}^{1 / 2},
\end{aligned}
$$

where $\left(x, p_{x}, y, p_{y}\right)$ denotes a representative phase point.

In analogy with the corresponding classical nonlinear dynamics study a generalized quantum Lyapunov exponent has also been defined ${ }^{18,20,23}$ as,

$$
\Lambda=\lim _{D(0) \rightarrow 0} \frac{1}{t \rightarrow \infty} \ln \left[\frac{D(t)}{D(0)}\right]
$$

and the corresponding Kolmogorov-Sinai-Lyapunov (KSL) entropy $\left(H^{Q u}\right)$ has been given by, ${ }^{18,20,23}$

$$
H^{Q u}=\sum_{\Lambda_{+} \succ 0} \Lambda_{+}
$$

\section{Numerical technique}

Classical Hamilton's equations of motion are solved using a fourth-order Runge-Kutta method. Once the phase points, $\left(x, p_{x}, y, p_{y}\right)$ are known at different time steps the phase space trajectories and the classical KSL entropy $\left(H^{C l}\right)$ are generated.

For the quantum problem the TDSE is solved by using a Peaceman-Rachford type finite difference algorithm ${ }^{25}$ using the following initial and boundary conditions:

$$
\begin{aligned}
& \psi(x, y, t=0)=\frac{1}{\sqrt{\pi}} \exp \left\{-\frac{1}{2}\left[\left(x-x_{o}\right)^{2}+\left(y-y_{o}\right)^{2}\right]\right\} \\
& \psi( \pm \infty, y, t)=0 \quad \forall y, t \\
& \psi(x, \pm \infty, t)=0 \quad \forall x, t
\end{aligned}
$$

The Guassian wavepacket is propagated for several time steps and for $-10 \leq x, y \leq 10$. Since the classical chaoticity requires more time to develop the classical solution is continued up to $2.56 \times 10^{3}$ time steps. All the quantities are in atomic units unless otherwise specified. The mesh sizes adopted are $\Delta x=\Delta y=$ $0 \cdot 08$. The temporal mesh sizes for the classical and quantum calculations are taken as $\Delta t=0.128$ and $\Delta t=0 \cdot 0128$ respectively.

After obtaining $\psi(x, y, t)$ by solving the TDSE, (4) are solved using a second-order Runge-Kutta method to generate the Bohmian trajectories which allow us to obtain the quantum KSL entropies $\left(H^{Q u}\right)$ by using (6) and (7). The initial separation of the trajectories is taken to be $10^{-5}$.

\section{Results and discussion}

The left and the right panels of the figure 1 respectively present the classical and quantal phase-portraits $\left(y, p_{y}\right)$ for four different field intensities. At $g=0$, a distinct torus is visible for the classical case. A cantorus like structure is obtained as the corresponding quantum analogue. As the field intensity increases the area of the phase space traversed by the trajectories increases and the system started exhibiting the typical Kolmogorov-Arnold-Moser type transition to the chaotic domain. In the quantum case also the corresponding transition is faithfully mimicked. In figure 1 we also present the classical and quantal phase space distance functions. For $g=0 \cdot 0,0 \cdot 1$ and $0 \cdot 5$ the initial small distance $\left(10^{-5}\right)$ of two nearby 

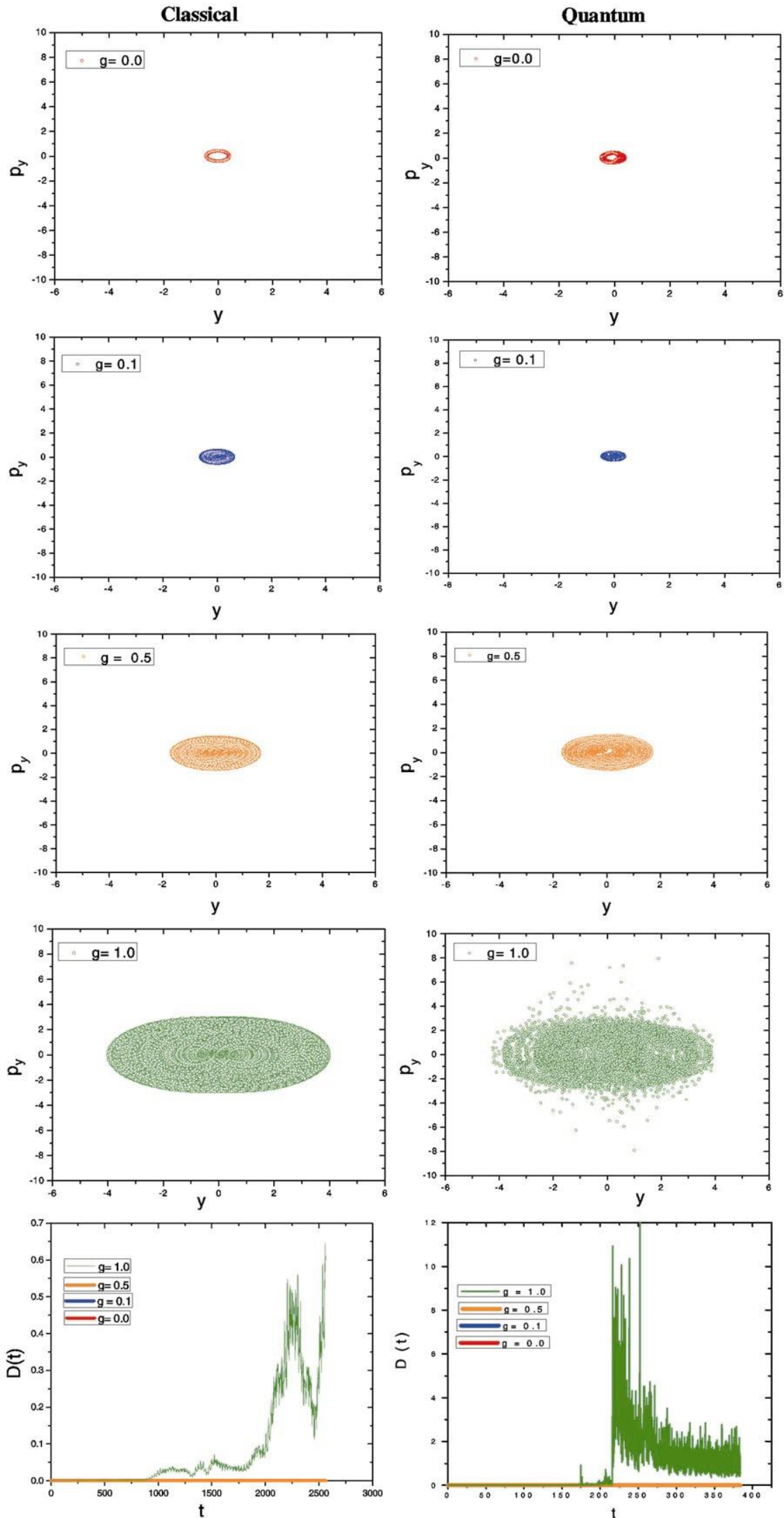

Figure 1. Classical and quantal phase portraits $\left(p_{y}\right.$ vs $\left.y\right)$ for the Henon-Heiles oscillator in the presence of an external field of varying intensities with $g=0 \cdot 0,0 \cdot 1,0 \cdot 5$ and $1 \cdot 0$. Also shown are the respective phase space distance functions $(D(t))$. 
quantum trajectories is more or less maintained which, however, drastically increases for higher field intensity $(g=1 \cdot 0)$. Once again the quantum stochasticity is manifested much earlier. The oscillation in $D(t)$ might have a bearing in the associated wavefunction revival. It deserves a careful scrutiny.

Time evolution of the classical KSL entropy $\left(H^{C l}\right)$ is depicted in the figure 2. As the system perturbation, originating from the external field, is increased

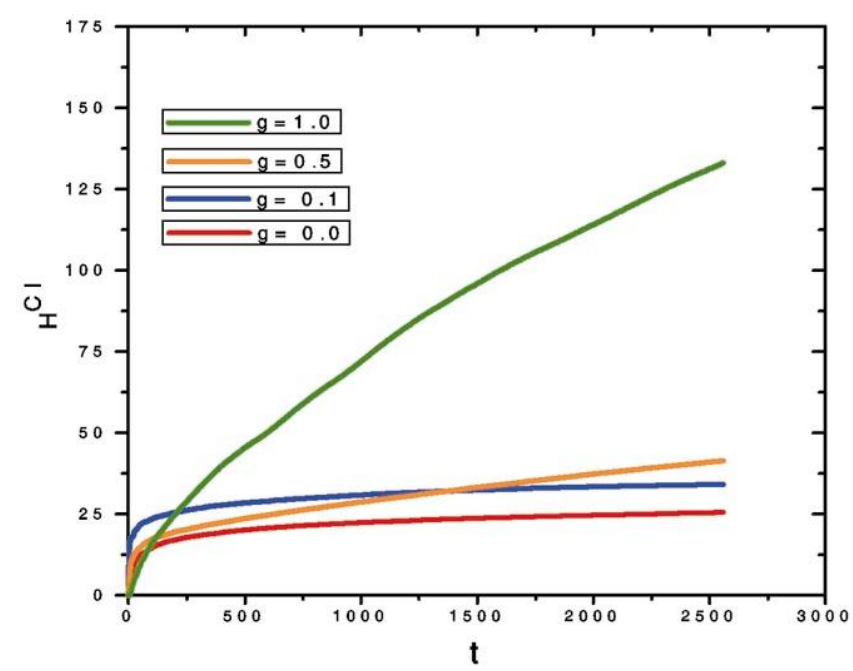

Figure 2. Time evolution of the KSL entropy associated with the classical motion $\left(H^{C l}\right)$ of the Henon-Heiles oscillator in the presence of an external field of varying intensities with $g=0 \cdot 0,0 \cdot 1,0 \cdot 5$ and $1 \cdot 0$.

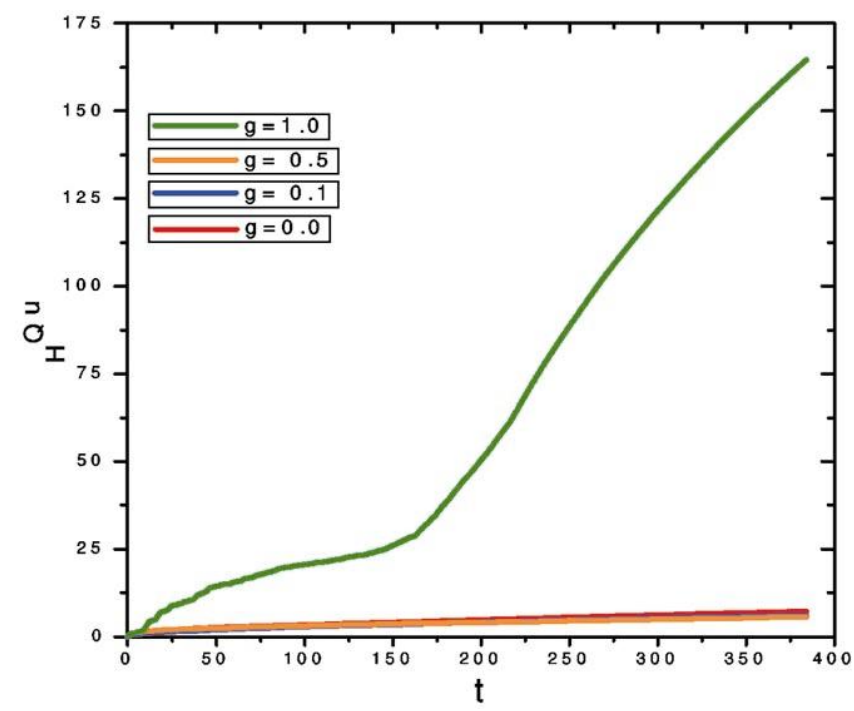

Figure 3. Time evolution of the KSL entropy associated with the quantal motion $\left(H^{Q u}\right)$ of the Henon-Heiles oscillator in the presence of an external field of varying intensities with $g=0 \cdot 0,0 \cdot 1,0 \cdot 5$ and $1 \cdot 0$. there results a discernible jump in the entropy value, a clear-cut signature of classical chaos through KAM transition.

Figure 3 presents the variation of the quantum KSL entropy $\left(H^{Q u}\right)$ as a function of time, for four different values of the external field intensity. It is interesting to note that there exists a one-to-one correspondence between the classical and quantum behaviour of the non-linear oscillator. The quantum signature of the classical chaos is conspicuous. Although the classical stochasticity enhances the quantum fluctuations $(g=1 \cdot 0)$, quantum non-classical effects somewhat suppress the classical chaos $(g=0 \cdot 0,0 \cdot 1,0 \cdot 5)$ as was observed ${ }^{18,20,23}$ in various non-linear dynamical systems.

\section{Concluding remarks}

Quantum signature of the Kolmogorov-Arnold-Moser transition of a Henon-Heiles oscillator in the presence of the external field of varying intensities is studied using the quantum theory of motion. A clearcut correspondence between the classical chaos and its quantum variant is observed in terms of the respective phase-portraits and the associated Kolmogorov-Sinai-Lyapunov entropies.

\section{Acknowledgements}

PKC would like to thank Professors V Krishnan and S Chandrasekaran for kindly inviting him to contribute in this special issue. We thank BRNS, Mumbai and CSIR, New Delhi for financial assistance.

\section{References}

1. Jensen R V 1995 Nature (London) 37316

2. Eckhardt B 1988 Phys. Rep. 163205

3. Gutzwiller M C 1990 Chaos in classical and quantum mechanics (New York: Springer-Verlag)

4. Reichl L E 1992 The transition to chaos in conservative classical systems: Quantum manifestations (New York: Springer-Verlag)

5. Henon M and Heiles C 1964 Astron. J. 6974

6. (a) Casati G, Chirikov B V Guaneri I and Shepelyansky D L 1978 Phys. Rep. 154 77; (b) Casati G Chiriko B V, Izrailev F M and Ford J 1979 In Stochastic behavior in classical and quantum Hamiltonian systems (eds) G Casati and J Ford (New York: Springer-Verlag) p. 375; (c) Casati G, Chirikov B V, Shepelyansky D L and Guarneri I 1986 Phys. Rev. Lett. 57823

7. Izrailev F M and Shepelyansky D L 1980 Theor. Math. Phys. 43553 
8. (a) Feit M D and Fleck Jr J A 1984 J. Chem. Phys. 80 2578; (b) Hanson J D, Cary J R and Meiss J D 1985 J. Stat. Phys. 39 327; (c) Meiss J D 1986 Parti. Accel. 19 9; (d) McKay R S and Meiss J D 1988 Phys. Rev. A: At. Mol. Opt. Phys. 37 4702; (e) Hanggi P 1993 In Activated barrier crossing (eds) G R Fleming and P Hanggi P (Singapore: World Scientific) p. 268

9. (a) Pomphrey N 1974 J. Phys. B7 1909; (b) de Polavieja G G 1996 Phys. Rev. A: At. Mol. Opt. Phys. 53 2059; 1996 Phys Lett A220 303; (c) Frisk H 1997 Phys. Lett. A227 139; (d) Konkel S and Makowski A 1998 J. Phys. Lett. A23895

10. (a) Swimm R T and Delos J B 1979 J. Chem. Phys. 71 1706; (b) Berry M V and Balazs N L $1979 \mathrm{~J}$. Phys. A12 625; (c) Hogg T and Huberman B A 1982 Phys. Rev. Lett. 48 711; (d) Grempel D R, Fishman S and Prange R E 1984 Phys. Rev. Lett. 53 1212; (e) Chang S and Shi K 1988 Phys. Rev. Lett. 55269

11. (a) Noid D W, Koszykowski M L, Tabor M and Marcus R A $1980 \mathrm{~J}$. Chem. Phys. 72 6169; (b) Nakamura K and Lakshmanan M 1986 Phys. Rev. Lett. 57 1661; (c) Nakamura K and Mikeska H J 1987 Phys. Rev. A: At. Mol. Opt. Phys. 35 5294; (d) Nakamura K and Thomas H 1988 Phys. Rev. Lett. 61 247; (e) Nakamura K, Bishop A R and Shudo A 1989 Phys. Rev. B: Condens. Matter 3912422

12. Noid D W, Koszykowski M L and Marcus R A 1979 J. Chem. Phys. 712864

13. (a) Davis M J and Heller E J 1979 J. Chem. Phys. 71 3383; (b) Davis M J, Stechel E B and Heller E J 1980 J. Chem. Phys. Lett. 76 21; (c) Davis M J and Heller E J 1981 J. Chem. Phys. 753916

14. Chattaraj P K 2000 Quantum theory of motion and quantum fluid dynamics of classically chaotic systems: An overview, in INSA special publication on Nonlinear Phenomena (eds) S K Malik, M K Chandrashekaran and N Pradhan (New Delhi: INSA Publication) p. 1047

15. (a) Chattaraj P K 1990 Nonlinear chemical dynamics, in Symmetries and singularity structures: Integrability and chaos, in Non-linear dynamical systems (eds) M Lakhshmanan and M Daniel (Berlin: SpringerVerlag) p 172; (b) Chattaraj P K, Sengupta S and Poddar A 1999 A quantum theory of motion for many electron systems within a quantum fluid density functional framework, in Non-linear dynamics and computational physics (ed.) VB Sheorey (New Delhi: Narosa,) p. 45

16. Nordholm K S and Rice S A 1974 J. Chem. Phys. 61 203768

17. (a) Hioe F T, MacMillen D and Montroll E W 1978 Phys. Rep. 43 305; (b) Hutchinson J S and Wyatt R E 1981 Phys. Rev. A: At. Mol. Opt. Phys. 23 1567; (c) Hioe F T and Singh S 1981 Phys. Rev. A: At. Mol. Opt. Phys. 242050

18. (a) Chattaraj P K and Sengupta S 1993 Phys. Lett. A181 225; (b) Sengupta S and Chattaraj P K 1996 Phys. Lett. A215 119; (c) Chattaraj P K and Sengupta S 1996 Indian J. Pure Appl. Phys. 34 518; (d) Chattaraj P K and Sengupta S 1996 Curr. Sci. 71 134; (e)
Chattaraj P K, Sengupta S and Poddar A 1998 Int. J. Quantum. Chem. DFT Spec. Issue 69 279; (f) Chattaraj P K, Maiti B and Sengupta S 2004 Int. J. Quantum Chem. 100254

19. Lin W A and Ballentine L E 1990 Phys. Rev. Lett. 65 2927

20. (a) Chattaraj P K and Sengupta S 1996 Curr. Sci. 71 134; (b) Chattaraj P K, Sengupta S and Poddar A 1998 Curr. Sci. 74 758; (c) Chattaraj P K, Sengupta S and Poddar A 1999 Curr. Sci. 76 1371; (d) Chattaraj P K, Sengupta S, Maiti B and Sarkar U 2002 Curr. Sci. 82 541; (e) Chattaraj P K and Maiti B 2002 Int. J. Mol. Sci. 3 338; (f) Chattaraj P K and Sarkar U 2003 Int. J. Quantum Chem. 91633

21. (a) Madelung E 1926 Z. Phys. 40 332; (b) de Broglie L 1926 C R Acad. Sci. 183 447; (c) Bohm D 1952 Phys. Rev. 85166 180; (d) Bohm D 1957 Causality and chance in modern physics (UK: Routledge); (e) Deb B M and Ghosh S K 1982 J. Chem. Phys. 77 342; (f) Bartolotti L 1982 J. Phys. Rev. A: At. Mol. Opt. Phys. 262243

22. (a) Holland P R 1993 The quantum theory of motion (Cambridge: Cambridge University Press); (b) Wyatt R E 2007 Quantum dynamics with trajectories: Introduction to quantum hydrodynamics (New York: Springer)

23. (a) Schwengelbeck U and Faisal F H M 1995 Phys. Lett. A199 281; (b) Faisal F H M and Schwengelbeck U 1995 Phys. Lett. A207 31; (c) Frisk H 1997 Phys. Lett. A227 139; (d) de Alcantara Bonfim O F, Florencio J and Sa'Barreto F C 1998 Phys. Rev. E: Stat. Phys. Plasmas Fluids Relat Interdiscip. Top 58 R2693; 2000 Phys. Lett. A27 129; (e) Wu H and Sprung D W L 1999 Phys. Lett. A261 150; (f) Makowski A J, Peplowski P and Dembiniski S T 2000 Phys. Lett. A266 241; (g) Cart-Wright J H E, Magnasco M O and Piro O 2002 Phys. Rev. E: Stat. Phys. Plasmas Fluids Relat Interdiscip. Top 65 069902; (h) Cart-Wright J H E, Magnasco M O and Piro O 2002 Phys. Rev. E: Stat. Phys. Plasmas. Fluids Relat. Interdiscip. Top $\mathbf{6 5}$ 045203; (i) Gouesbet G, Meunier-Guttin-Cluzel S and Grehan G 2002 Phys. Rev. E: Stat. Phys. Plasmas. Fluids Relat. Interdiscip. Top 65 016212; (j) LunaAcosta G A, Méndez-Bermúdez A, Seba $P$ and Pichugin K N 2002 Phys. Rev. E65 046605

24. (a) Lopreore C L and Wyatt R E 1999 Phys. Rev. Lett. 82 5190; (b) Sanz A S, Borondo F and MiretArtes S 2000 Phys. Rev. B: Condens. Matter 61 7743; (c) Prezhdo O V and Brooksby C 2001 Phys. Rev. Lett. 86 3215; (d) Grübl G, Moser R and Rheinberger K 2001 J. Phys. A: Math. Gen. Phys. 34 2753; (e) Gindensperger E, Meier C and Beswick J A $2002 \mathrm{~J}$. Chem. Phys. 113 9369; Gindensperger E, Meier C and Beswick J A $2002 \mathrm{~J}$. Chem. Phys. 116 8; (f) Colijn C and Vrscay E R 2002 Phys. Lett. A300 334

25. (a) Goldberg A, Schey H M and Schwartz J L 1967 Am. J. Phys. 35 177; 1968 Am. J. Phys. 36 454; (b) Galbraith I, Ching Y S and Abraham E 1984 Am. J. Phys. 52 60; (c) Chattaraj P K, Rao K S and Deb B M 1987 J. Comput. Phys. 72504 